\title{
BKM's criterion for the 3D nematic liquid crystal flows in Besov spaces of negative regular index
}

\section{Baoquan Yuan*, Chengzhou Wei}

School of Mathematics and Information Science, Henan Polytechnic University, Henan, 454000, China.

\author{
Communicated by Y. Hu
}

\begin{abstract}
In this paper, we investigate the blow-up criterion of a smooth solution of the nematic liquid crystal flow in threedimensional space. More precisely, We prove that if $\int_{0}^{T}\left(\|\omega\|_{\dot{B}_{\infty, \infty}^{-\alpha}}^{\frac{2}{2-\alpha}}+\|\nabla d\|_{\dot{B}_{\infty, \infty}^{0}}^{2}\right) d t<\infty, 0<\alpha<2$, then the solution $(u, d)$ can be extended smoothly beyond $t=T$. (C) 2017 All rights reserved.
\end{abstract}

Keywords: Nematic liquid crystal flow, blow-up criteria, regularity criteria, Besov space. 2010 MSC: 35Q35, 35B65.

\section{Introduction}

In this paper, we are concerned with the following viscous incompressible flow of nematic liquid crystal in $\mathbb{R}^{3}$ :

$$
\left\{\begin{array}{l}
\frac{\partial u}{\partial t}+u \cdot \nabla u-\mu \Delta u+\nabla p=-\lambda \nabla \cdot(\nabla d \odot \nabla d) \\
\frac{\partial d}{\partial t}+u \cdot \nabla d=\gamma\left(\Delta d+|\nabla d|^{2} d\right) \\
\operatorname{divu}=0 \\
u(x, 0)=u_{0}, d(x, 0)=d_{0} .
\end{array}\right.
$$

Here $u=\left(u_{1}(x, t), u_{2}(x, t), u_{3}(x, t)\right)$ denotes the velocity of the fluid at a point $x \in \mathbb{R}^{3}$ and time $t \in[0, T)$; and $d=\left(d_{1}(x, t), d_{2}(x, t), d_{3}(x, t)\right)$ and $p=p(x, t)$ stand for the macroscopic average of the nematic liquid crystal orientation field and the fluid pressure, respectively. The tensorial notation $\nabla \mathrm{d} \odot \nabla \mathrm{d}$ denotes the $3 \times 3$ matrix whose $(i, j)$-th entry is given by $\partial_{x_{i}} d \cdot \partial_{x_{j}} d$, and then $(\nabla d \odot \nabla d)_{i j}=\sum_{k=1}^{3} \partial x_{i} d_{k} \partial x_{j} d_{k}$ for any $i, j=1,2,3$. Moreover, it is easy to verify that $\nabla \cdot(\nabla \mathrm{d} \odot \nabla \mathrm{d})=\frac{1}{2} \nabla\left(|\nabla \mathrm{d}|^{2}\right)+\Delta \mathrm{d} \cdot \nabla \mathrm{d}$, where $\nabla$ denotes the gradient operator $\left(\partial_{x_{1}}, \partial_{x_{2}}, \partial_{x_{3}}\right) . u_{0}$ and $d_{0}$ are the prescribed initial data of $u$ and $d$, and $u_{0}$ satisfies the incompressible condition divu $u_{0}=0$. Clearly $\Delta|\mathrm{d}|^{2}=0$ because of $|\mathrm{d}|=1$, we thus have $\mathrm{d} \cdot \Delta \mathrm{d}=-|\nabla \mathrm{d}|^{2}$.

\footnotetext{
*Corresponding author

Email addresses: bqyuan@hpu .edu.cn (Baoquan Yuan), jisuanwei@163.com (Chengzhou Wei)
} 
$\mu$ is the kinematic viscosity, $\lambda$ is the competition between the kinetic and potential energies, and $\gamma$ is the microscopic elastic relation time for the molecular orientation field.

The system (1.1) is a simplified version of the Ericksen-Leslie model (see [5, 16]), which was first introduced by Lin in [18] and studied by Lin and Liu in their important works in [21, 22] and Coutand and Shkoller in [4]. It is a macroscopic continuum description of the time evolution of the rod-like liquid crystal materials under the influence of both the velocity field $u$ and the macroscopic description of the microscopic orientation configurations d. Due to the physical importance and real-world applications, there have been numerous attempts to formulate continuum theories describing the behavior of liquid crystal flows, we refer to the seminal papers $[5,16]$ and references therein. Mathematically, the system (1.1) can be seen as a variant of the Navier-Stokes problem with respect to the unknown velocity and pressure $(u, p)$ coupled with heat flow of a harmonic map. When $d$ is constant, this model becomes the well-known Navier-Stokes equation, we refer to the papers $[1,8,13,14]$, while when $u=0$, the system is the harmonic heat flow equation on to a sphere [3, 28].

Besides the physical and real-world applications, there have been numerous attempts to study it from mathematical viewpoint. Many results to system (1.1) have been established (see, for example, [7, 9$11,17,19,20,23,24,26,27,29])$. Lin et al. [20] proved the global existence of Leray-Hopf type weak solutions for system (1.1) on the bounded domain in $\mathbb{R}^{2}$ under initial and boundary value conditions. When the space dimension $\mathrm{n} \geqslant 3, \mathrm{Li}$ and Wang [17] obtained the existence of a local strong solution for general initial data and the global strong solution for small initial data. It is well-known that the strong solutions of heat flow of harmonic maps must be blowing up at finite time (see [2]), therefore we can not expect that the system (1.1) has a global smooth solution with general initial data. However, Wen and Ding [29] proved that if $u_{0}(x) \in H^{s}\left(\mathbb{R}^{3}, \mathbb{R}^{3}\right)$ with $\nabla \cdot u=0$, and $d_{0} \in H^{s+1}\left(\mathbb{R}^{3}, S^{2}\right)$ for $s \geqslant 3$, then there exists $T=T\left(\left\|u_{0}\right\|_{H^{s}},\left\|d_{0}\right\|_{H^{s+1}}\right)$ such that system (1.1) has a unique local classical solution in the class

$$
\left\{\begin{array}{l}
u \in C\left([0, T] ; H^{s}\left(\mathbb{R}^{3}, \mathbb{R}^{3}\right)\right) \cap C^{1}\left([0, T] ; H^{s-2}\left(\mathbb{R}^{3}, \mathbb{R}^{3}\right)\right), \\
d \in C\left([0, T] ; H^{s+1}\left(\mathbb{R}^{3}, S^{2}\right)\right) \cap C^{1}\left([0, T] ; H^{s-1}\left(\mathbb{R}^{3}, S^{2}\right)\right) .
\end{array}\right.
$$

The blow-up criteria of smooth solutions to nematic liquid crystal flow are important topic in the research of global well-posedness. Huang and Wang [11] established a BKM type blow-up criterion for the system (1.1). Namely, if $\mathrm{T}^{*}$ is the maximal time, $0<\mathrm{T}^{*}<+\infty$, then

$$
\int_{0}^{T^{*}}\left(\|\omega\|_{\infty}+\|\nabla \mathrm{d}\|_{\infty}^{2}\right) \mathrm{dt}=\infty .
$$

This result is improved by Liu and Zhao [25], who proved that the smooth solution (u,d) of (1.1) blows up at the time $\mathrm{T}^{*}$, if and only if

$$
\int_{0}^{\mathrm{T}^{*}} \frac{\|\omega\|_{\dot{\mathrm{B}}_{\infty, \infty}^{0}}+\|\nabla \mathrm{d}\|_{\dot{\mathrm{B}}_{\infty, \infty}^{0}}^{2}}{\sqrt{1+\ln \left(\mathrm{e}+\|\omega\|_{\dot{\mathrm{B}}_{\infty, \infty}^{0}}+\|\nabla \mathrm{d}\|_{\dot{\mathrm{B}}_{\infty, \infty}^{0}}\right)}} \mathrm{dt}=\infty .
$$

This result is also improved by Zhao [30] in terms of two velocity components and molecular orientations. More precisely, let $T^{*}$ be the maximal existence time of the local strong solution $(u, d)$, then $T^{*}<+\infty$ if and only if

$$
\int_{0}^{T^{*}}\left(\left\|\nabla_{\mathrm{h}} \mathrm{u}^{\mathrm{h}}\right\|_{\dot{\mathrm{B}}_{\mathrm{p}, 2 \mathrm{p} / 3}^{0}}^{\mathrm{q}}+\|\nabla \mathrm{d}\|_{\dot{\mathrm{B}}_{\infty, \infty}^{0}}^{2}\right) \mathrm{dt}=\infty, \text { with } 3 / \mathrm{p}+2 / \mathrm{q}=2,3 / 2<\mathrm{p}<\infty .
$$

Recently, a new blow-up criterion in terms of velocity gradient in Besov spaces of negative indices is established by Fan and Zhou [6], i.e.,

$$
\nabla \mathrm{u}, \Delta^{2} \mathrm{~d} \in \mathrm{L}^{\frac{2}{2-\alpha}}\left(0, \mathrm{~T} ; \dot{\mathrm{B}}_{\infty, \infty}^{-\alpha}\right), \quad \text { with } 0<\alpha<1 .
$$

In this paper, we consider the blow-up criterion of system (1.1) in terms of the vorticity in Besov space of negative index and the orientation field in the homogeneous Besov space, which improves the result of $[11,25]$. 
Before stating our main result, we introduce some function spaces and notations. The norm of the Lebesgue space $\|\cdot\|_{L^{p}}$ is denoted by $\|\cdot\|_{p}$. To simplify the notations, we shall use the letter $C$ to denote a generic constant which may vary from line to line, and write $\partial_{t} u=\frac{\partial u}{\partial t}, \partial_{i}=\frac{\partial}{\partial x_{i}},(u \cdot \nabla)=\sum_{i=1}^{3} u_{i} \partial_{i}$. Since the concrete values of the constants $\mu, \lambda, \gamma$ play no role in our discussion, to simplify the presentation, we shall assume that $\mu=\lambda=\gamma=1$ in this paper.

To this end, we state our result as follows.

Theorem 1.1. Let $\mathrm{u}_{0} \in \mathrm{H}^{\mathrm{s}}\left(\mathbb{R}^{3}, \mathbb{R}^{3}\right)$ with $\nabla \cdot \mathrm{u}_{0}=0$ and $\mathrm{d}_{0} \in \mathrm{H}^{\mathrm{s}+1}\left(\mathbb{R}^{3}, \mathrm{~S}^{2}\right)$ with $\mathrm{s} \geqslant 3$. Suppose that the pair $(u, p)$ is a smooth solution to the equations (1.1) satisfying (1.2). If $(u, d)$ satisfies the condition

$$
\int_{0}^{T}\left(\|\omega(\mathrm{t})\|_{\dot{\mathrm{B}}_{\infty, \infty}^{-r}}^{\frac{2}{2-\mathrm{r}}}+\|\nabla \mathrm{d}(\mathrm{t})\|_{\dot{\mathrm{B}}_{\infty, \infty}^{0}}^{2}\right) d \mathrm{t}<\infty, \quad 0<\mathrm{r}<2,
$$

then the solution $(\mathrm{u}, \mathrm{d})$ can be extended smoothly beyond $\mathrm{t}=\mathrm{T}$.

\section{Preliminaries}

In this preliminary section, we will present some lemmas which will be used in the proof of Theorem 1.1 .

Lemma 2.1 ([1, Theorem 2.42]). Let $1 \leqslant \mathrm{q}<\mathrm{p}<\infty$ and $\alpha$ be a positive real number. Then there exists a constant C such that

$$
\|f\|_{L^{p}} \leqslant C\|f\|_{\dot{B}_{\infty}^{-\alpha, \infty}}^{1-\theta}\|f\|_{\dot{\mathrm{B}}_{q, q}^{\beta}, \mathfrak{q}^{\prime}}^{\theta} \text { with } \beta=\alpha\left(\frac{p}{\mathrm{q}}-1\right) \text { and } \theta=\frac{\mathrm{q}}{\mathrm{p}} \text {. }
$$

In particular, for $q=2$ and $p=3$, we have

$$
\|\mathbf{f}\|_{L^{3}}^{3} \leqslant C\|f\|_{\dot{B}_{\infty, \infty}^{-r}}\|f\|_{\dot{H}^{\frac{r}{2}}}^{2}, \quad \text { with } r>0 .
$$

For $p=4, q=2, \alpha=\beta=1$, we have

$$
\|\mathrm{f}\|_{4} \leqslant \mathrm{C}\|\mathrm{f}\|_{\dot{\mathrm{B}}_{\infty, \infty}^{-1}}^{\frac{1}{2}}\|\nabla \mathrm{f}\|_{2}^{\frac{1}{2}}
$$

Lemma 2.2 (Commutator estimate [12]). Let $1<p<\infty, s>0$. Assume that $f \in \dot{W}^{1, p_{1}} \cap \dot{W}^{s, p_{3}}$ and $\mathrm{g} \in \mathrm{L}^{\mathrm{p}_{4}} \cap \dot{\mathrm{W}}^{\mathrm{s}-1, \mathrm{p}_{2}}$, then there exists constant $\mathrm{C}$ independent of $\mathrm{f}, \mathrm{g}$ such that

$$
\left\|\wedge^{s}(f g)-f \wedge^{s} g\right\|_{p} \leqslant C\left(\|\nabla f\|_{p_{1}}\|g\|_{\dot{W}^{s-1, p_{2}}}+\|f\|_{\dot{W}^{s, p_{3}}}\|g\|_{p_{4}}\right)
$$

with $\mathrm{p}_{2}, \mathrm{p}_{3} \in(1,+\infty)$ such that

$$
\frac{1}{p}=\frac{1}{p_{1}}+\frac{1}{p_{2}}=\frac{1}{p_{3}}+\frac{1}{p_{4}} .
$$

Here $\wedge:=(-\Delta)^{\frac{1}{2}}$ is defined through the Fourier transform, namely

$$
\widehat{\wedge f}(\xi)=|\xi| \hat{f}(\xi) .
$$

\section{Proof of Theorem 1.1}

In this section we devote to prove Theorem 1.1, and the proof will be divided into two steps.

Step I. $\|\mathrm{u}\|_{\mathrm{H}^{1}}+\|\nabla \mathrm{d}\|_{\mathrm{H}^{1}}$ estimate.

In this step, we will show:

$$
\sup _{0<\mathrm{t}<\mathrm{T}}\left(\|\nabla \mathrm{u}\|_{2}^{2}+\|\Delta \mathrm{d}\|_{2}^{2}\right) \leqslant \mathrm{C} .
$$

Firstly, we estimate $L^{2}$ norms of $u$ and $\nabla d$. Multiplying both sides of the first equation of (1.1) by $u$ and 
integrating over $\mathbb{R}^{3}$, and then applying the identity $\nabla \cdot(\nabla \mathrm{d} \odot \nabla \mathrm{d})=\frac{1}{2} \nabla\left(|\nabla \mathrm{d}|^{2}\right)+\Delta \mathrm{d} \cdot \nabla \mathrm{d}$, we obtain

$$
\frac{1}{2} \frac{\mathrm{d}}{\mathrm{dt}}\|\mathrm{u}\|_{2}^{2}+\|\nabla \mathrm{u}\|_{2}^{2}=-\int_{\mathbb{R}^{3}} \nabla \mathrm{d} \cdot \Delta \mathrm{d} \cdot \mathrm{udx},
$$

where we have used the fact divu $=0$. Multiplying both sides of the second equation of (1.1) by $-\Delta \mathrm{d}$ and integrating over $\mathbb{R}^{3}$, one has

$$
\frac{1}{2} \frac{\mathrm{d}}{\mathrm{dt}}\|\nabla \mathrm{d}\|_{2}^{2}+\|\Delta \mathrm{d}\|_{2}^{2}-\int_{\mathbb{R}^{3}}(\mathrm{u} \cdot \nabla \mathrm{d}) \cdot \Delta \mathrm{dd} x=-\int_{\mathbb{R}^{3}}|\nabla \mathrm{d}|^{2} \mathrm{~d} \cdot \Delta \mathrm{dd} x=\int_{\mathbb{R}^{3}}|\mathrm{~d} \cdot \Delta \mathrm{d}|^{2} \mathrm{~d} x \leqslant \int_{\mathbb{R}^{3}}|\Delta \mathrm{d}|^{2} \mathrm{~d} x,
$$

where we have used the facts $|\mathrm{d}|=1$ and $|\nabla d|^{2}=-\mathrm{d} \cdot \Delta \mathrm{d}$. Combining (3.2) and (3.3), and integrating in time, we get

$$
\sup _{0<\mathrm{t}<\mathrm{T}}\left(\|\mathrm{u}\|_{2}^{2}+\|\nabla \mathrm{d}\|_{2}^{2}\right)+\int_{0}^{\mathrm{T}}\|\nabla \mathrm{u}\|_{2}^{2} \mathrm{dt} \leqslant\left\|\mathrm{u}_{0}\right\|_{2}^{2}+\left\|\nabla \mathrm{d}_{0}\right\|_{2}^{2} .
$$

Secondly, taking the inner product of $-\Delta u$ with the first equation of (1.1), by integrating by parts and using the incompressibility condition, we have

$$
\frac{1}{2} \frac{\mathrm{d}}{\mathrm{dt}}\|\nabla \mathrm{u}\|_{2}^{2}+\|\Delta \mathrm{u}\|_{2}^{2} \leqslant \int_{\mathbb{R}^{3}}(\mathrm{u} \cdot \nabla) \mathrm{u} \cdot \Delta \mathrm{udx}+\int_{\mathbb{R}^{3}} \nabla \mathrm{d} \cdot \Delta \mathrm{d} \cdot \Delta \mathrm{ud} x
$$

For the estimate of $\Delta \mathrm{d}$, taking $\Delta$ on both sides of the second equation of (1.1), multiplying $\Delta \mathrm{d}$ and integrating over $\mathbb{R}^{3}$, one has

$$
\frac{1}{2} \frac{\mathrm{d}}{\mathrm{dt}}\|\Delta \mathrm{d}\|_{2}^{2}+\|\nabla \Delta \mathrm{d}\|_{2}^{2} \leqslant-\int_{\mathbb{R}^{3}} \Delta(\mathrm{u} \cdot \nabla \mathrm{d}) \cdot \Delta \mathrm{dd} x+\int_{\mathbb{R}^{3}} \Delta\left(|\nabla \mathrm{d}|^{2} \mathrm{~d}\right) \cdot \Delta \mathrm{dd} x
$$

Combining the estimates (3.4) and (3.5), we obtain that

$$
\begin{aligned}
\frac{1}{2} \frac{\mathrm{d}}{\mathrm{dt}}\left(\|\nabla \mathrm{u}\|_{2}^{2}+\|\Delta \mathrm{d}\|_{2}^{2}\right)+\|\Delta \mathrm{u}\|_{2}^{2}+\|\nabla \Delta \mathrm{d}\|_{2}^{2} \leqslant & \int_{\mathbb{R}^{3}} \mathrm{u} \cdot \nabla \mathrm{u} \cdot \Delta \mathrm{udx}+\int_{\mathbb{R}^{3}} \nabla \mathrm{d} \cdot \Delta \mathrm{d} \cdot \Delta \mathrm{ud} x \\
& -\int_{\mathbb{R}^{3}} \Delta(\mathrm{u} \cdot \nabla \mathrm{d}) \cdot \Delta \mathrm{d} \mathrm{d} x+\int_{\mathbb{R}^{3}} \Delta\left(|\nabla \mathrm{d}|^{2} \mathrm{~d}\right) \cdot \Delta \mathrm{d} \mathrm{d} x \\
& =\mathrm{I}_{1}+\mathrm{I}_{2}+\mathrm{I}_{3}+\mathrm{I}_{4} .
\end{aligned}
$$

In what follows, we will deal with each term on the right-hand side of (3.6) separately. The first term can be bounded by (2.1) as follows.

$$
\begin{aligned}
\mathrm{I}_{1}=\int_{\mathbb{R}^{3}} \mathrm{u} \cdot \nabla \mathrm{u} \cdot \Delta \mathrm{udx} & \leqslant-\sum_{k=1}^{3} \int_{\mathbb{R}^{3}} \partial_{\mathrm{k}} \mathrm{u} \cdot \nabla \mathrm{u} \cdot \partial_{\mathrm{k}} \mathrm{udx} \\
& \leqslant \mathrm{C}\|\omega\|_{3}^{3} \leqslant \mathrm{C}\|\omega\|_{\dot{\mathrm{B}}_{\infty, \infty}^{-r}}^{3}\|\omega\|_{\dot{H}^{\frac{r}{2}}}^{2} \\
& \leqslant \mathrm{C}\|\omega\|_{\dot{\mathrm{B}}_{\infty, \infty}^{-r}}^{-r}\|\nabla u\|_{2}^{2-r}\|\nabla \omega\|_{2}^{\mathrm{r}} \\
& \leqslant \mathrm{C}\|\omega\|_{\dot{\mathrm{B}}_{\infty, \infty}^{-r}}^{2-r}\|\nabla u\|_{2}^{2}+\frac{1}{6}\|\Delta \mathrm{u}\|_{2}^{2} .
\end{aligned}
$$

To estimate $I_{2}, I_{3}, I_{4}$, we use (2.2) with $f=\Delta d$ and $|d|=1$, Young inequality and Hölder inequality and the following Gagliardo-Nirenberg inequality:

$$
\|\nabla \mathrm{d}\|_{4}^{2} \leqslant\|\mathrm{~d}\|_{\infty}\|\Delta \mathrm{d}\|_{2}
$$




$$
\begin{aligned}
\mathrm{I}_{2}=\int_{\mathbb{R}^{3}} \nabla \mathrm{d} \cdot \Delta \mathrm{d} \cdot \Delta \mathrm{ud} x & \leqslant\|\Delta \mathrm{u}\|_{2}\|\nabla \mathrm{d}\|_{4}\|\Delta \mathrm{d}\|_{4} \\
& \leqslant \frac{1}{6}\|\Delta \mathrm{u}\|_{2}^{2}+\mathrm{C}\|\mathrm{d}\|_{\infty}\|\Delta \mathrm{d}\|_{2}\|\nabla \mathrm{d}\|_{\dot{\mathrm{B}}_{\infty, \infty}^{0}}\|\nabla \Delta \mathrm{d}\|_{2} \\
& \leqslant \frac{1}{6}\|\Delta \mathrm{u}\|_{2}^{2}+\frac{1}{6}\|\nabla \Delta \mathrm{d}\|_{2}^{2}+\mathrm{C}\|\nabla \mathrm{d}\|_{\dot{\mathrm{B}}_{\infty, \infty}^{0}}^{2}\|\Delta \mathrm{d}\|_{2}^{2} \\
\mathrm{I}_{3}=-\int_{\mathbb{R}^{3}} \Delta(\mathrm{u} \cdot \nabla \mathrm{d}) \Delta \mathrm{dd} x & \leqslant \int_{\mathbb{R}^{3}}|\Delta \mathrm{u}\|\nabla \mathrm{d}\| \Delta \mathrm{d}| \mathrm{d} x+2 \int_{\mathbb{R}^{3}}\left|\nabla \mathrm{u}\left\|\nabla^{2} \mathrm{~d}\right\| \Delta \mathrm{d}\right| \mathrm{d} x \\
& \leqslant \mathrm{C}\left(\|\Delta \mathrm{u}\|_{2}\|\nabla \mathrm{d}\|_{4}\|\Delta \mathrm{d}\|_{4}+\|\nabla \mathrm{u}\|_{2}\left\|\nabla^{2} \mathrm{~d}\right\|_{4}\|\Delta \mathrm{d}\|_{4}\right) \\
& \leqslant \mathrm{C}\|\Delta \mathrm{u}\|_{2}\|\mathrm{~d}\|_{\infty}^{\frac{1}{2}}\|\Delta \mathrm{d}\|_{2}^{\frac{1}{2}}\|\nabla \mathrm{d}\|_{\dot{\mathrm{B}}_{\infty, \infty}^{0}}^{\frac{1}{2}}\|\nabla \Delta \mathrm{d}\|_{2}^{\frac{1}{2}}+\|\nabla \mathrm{u}\|_{2}\|\nabla \mathrm{d}\|_{\dot{\mathrm{B}}_{\infty, \infty}^{0}}\|\nabla \Delta \mathrm{d}\|_{2} \\
& \leqslant \frac{1}{6}\|\Delta \mathrm{u}\|_{2}^{2}+\frac{1}{6}\|\nabla \Delta \mathrm{d}\|_{2}^{2}+\mathrm{C}\|\nabla \mathrm{d}\|_{\dot{\mathrm{B}}_{\infty, \infty}^{0}}^{2}\left(\|\nabla \mathrm{u}\|_{2}^{2}+\|\Delta \mathrm{d}\|_{2}^{2}\right),
\end{aligned}
$$

where we have used the fact that divu $=0$ implies that $\int_{\mathbb{R}^{3}} u \cdot \nabla \Delta d \cdot \Delta d d x=0$.

$$
\begin{aligned}
\mathrm{I}_{4}=\int_{\mathbb{R}^{3}} \Delta\left(|\nabla \mathrm{d}|^{2} \mathrm{~d}\right) \Delta \mathrm{d} \mathrm{d} x & \leqslant\left.\left|\int_{\mathbb{R}^{3}} \nabla \mathrm{d}\right| \nabla \mathrm{d}\right|^{2} \cdot \nabla \Delta \mathrm{d} \mathrm{d} x|+| \int_{\mathbb{R}^{3}} \mathrm{~d} \nabla\left(|\nabla \mathrm{d}|^{2}\right) \cdot \nabla \Delta \mathrm{d} \mathrm{d} x \mid \\
& \leqslant\left|\int_{\mathbb{R}^{3}} \nabla\left(\nabla \mathrm{d}|\nabla \mathrm{d}|^{2}\right) \Delta \mathrm{d} \mathrm{d} x\right|+\left|\int_{\mathbb{R}^{3}} \mathrm{~d} \nabla\left(|\nabla \mathrm{d}|^{2}\right) \cdot \nabla \Delta \mathrm{d} \mathrm{d} x\right| \\
& \leqslant\left.\mathrm{C}\left|\int_{\mathbb{R}^{3}}\right| \nabla \mathrm{d}\right|^{2}\left|\nabla^{2} \mathrm{~d}\right|^{2} \mathrm{~d} x|+\mathrm{C}| \int_{\mathbb{R}^{3}}\left|\nabla \mathrm{d} \| \nabla^{2} \mathrm{~d}\right||\nabla \Delta \mathrm{d}| \mathrm{d} x \mid \\
& \leqslant \mathrm{C}\|\nabla \mathrm{d}\|_{4}^{2}\left\|\nabla^{2} \mathrm{~d}\right\|_{4}^{2}+\mathrm{C}\|\nabla \mathrm{d}\|_{4}\left\|\nabla^{2} \mathrm{~d}\right\|_{4}\|\nabla \Delta \mathrm{d}\|_{2} \\
& \leqslant \frac{1}{12}\|\nabla \Delta \mathrm{d}\|_{2}^{2}+\mathrm{C}\|\nabla \mathrm{d}\|_{4}^{2}\left\|\nabla^{2} \mathrm{~d}\right\|_{4}^{2} \\
& \leqslant \frac{1}{6}\|\nabla \Delta \mathrm{d}\|_{2}^{2}+\mathrm{C}\|\nabla \mathrm{d}\|_{\dot{\mathrm{B}}_{\infty, \infty}^{0}}^{2}\|\Delta \mathrm{d}\|_{2}^{2} .
\end{aligned}
$$

Plugging the estimates (3.7)-(3.10) back into (3.6) and absorbing the diffusive terms, one thus can deduce

$$
\frac{\mathrm{d}}{\mathrm{dt}}\left(\|\nabla \mathrm{u}\|_{2}^{2}+\|\Delta \mathrm{d}\|_{2}^{2}\right)+\frac{1}{2}\left(\|\Delta \mathrm{u}\|_{2}^{2}+\|\nabla \Delta \mathrm{d}\|_{2}^{2}\right) \leqslant \mathrm{C}\left(\|\omega\|_{\dot{\mathrm{B}}_{\infty, \infty}^{-r}}^{\frac{2}{2-r}}+\|\nabla \mathrm{d}\|_{\dot{\mathrm{B}}_{\infty, \infty}^{0}}^{2}\right)\left(\|\nabla \mathrm{u}\|_{2}^{2}+\|\Delta \mathrm{d}\|_{2}^{2}\right) .
$$

By Gronwall's inequality, we have

$$
\begin{aligned}
& \|\nabla \mathrm{u}\|_{2}^{2}+\|\Delta \mathrm{d}\|_{2}^{2}+\int_{0}^{\mathrm{T}}\left(\|\Delta \mathrm{u}\|_{2}^{2}+\|\nabla \Delta \mathrm{d}\|_{2}^{2}\right) \mathrm{dt} \\
& \quad \leqslant\left(\left\|\nabla \mathrm{u}_{0}\right\|_{2}^{2}+\left\|\Delta \mathrm{d}_{0}\right\|_{2}^{2}\right) \exp \left\{\int_{0}^{\mathrm{T}}\left(\|\omega\|_{\dot{\mathrm{B}}_{\infty, \infty}^{-r}}^{\frac{2}{2-r}}+\|\nabla \mathrm{d}\|_{\dot{\mathrm{B}}_{\infty, \infty}^{0}}^{2}\right) \mathrm{d} \mathrm{t}\right\}<\mathrm{C} .
\end{aligned}
$$

Step II. $\|\mathrm{u}\|_{\mathrm{H}^{2}}+\|\nabla \mathrm{d}\|_{\mathrm{H}^{2}}$ estimate.

In the following, we will show how to deduce $\|u\|_{H^{2}}+\|\nabla \mathrm{d}\|_{\mathrm{H}^{2}}$ estimate from $\|u\|_{\mathrm{H}^{1}}+\|\nabla \mathrm{d}\|_{\mathrm{H}^{1}}$ estimate obtained in Step I.

Taking the operation $\nabla^{2}$ on both sides of the first equation of (1.1), $\nabla^{3}$ on both sides of the second equation of (1.1), multiplying $\left(\nabla^{2} u, \nabla^{3} \mathrm{~d}\right)$ to the resulting equations, and integrating over $\mathbb{R}^{3}$, we get

$$
\begin{aligned}
\frac{1}{2} \frac{\mathrm{d}}{\mathrm{dt}}\left(\left\|\nabla^{2} \mathrm{u}\right\|_{2}^{2}+\left\|\nabla^{3} \mathrm{~d}\right\|_{2}^{2}\right)+\left\|\nabla^{3} \mathrm{u}\right\|_{2}^{2}+\left\|\nabla^{4} \mathrm{~d}\right\|_{2}^{2} & \leqslant-\int_{\mathbb{R}^{3}} \nabla^{2}(\mathrm{u} \cdot \nabla \mathrm{u}) \nabla^{2} \mathrm{ud} x+\int_{\mathbb{R}^{3}} \nabla^{2}(\nabla \mathrm{d} \cdot \Delta \mathrm{d}) \nabla^{2} \mathrm{ud} x \\
& -\int_{\mathbb{R}^{3}} \nabla^{3}(\mathrm{u} \cdot \nabla \mathrm{d}) \nabla^{3} \mathrm{dd} x+\int_{\mathbb{R}^{3}} \nabla^{3}\left(|\nabla \mathrm{d}|^{2} \mathrm{~d}\right) \nabla^{3} \mathrm{dd} x \\
& =\mathrm{J}_{1}+\mathrm{J}_{2}+\mathrm{J}_{3}+\mathrm{J}_{4} .
\end{aligned}
$$

We estimate the right-hand side of (3.11) as follows.

For the first term, the commutator estimate (2.3) together with Gagliardo-Nirenberg inequality and Hölder inequality yield that 


$$
\begin{aligned}
\mathrm{J}_{1} & =-\int_{\mathbb{R}^{3}}\left[\nabla^{2}(\mathrm{u} \cdot \nabla \mathrm{u})-(\mathrm{u} \cdot \nabla) \nabla^{2} \mathrm{u}\right] \nabla^{2} \mathrm{udx} \\
& \leqslant \mathrm{C}\|\nabla \mathrm{u}\|_{2}\left\|\nabla^{2} \mathrm{u}\right\|_{4}^{2} \\
& \leqslant \mathrm{C}\|\nabla \mathrm{u}\|_{2}\left\|\nabla^{2} \mathrm{u}\right\|_{2}^{\frac{1}{2}}\left\|\nabla^{3} \mathrm{u}\right\|_{2}^{\frac{3}{2}} \\
& \leqslant \mathrm{C}\|\nabla \mathrm{u}\|_{2}^{4}\left\|\nabla^{2} \mathrm{u}\right\|_{2}^{2}+\frac{1}{6}\left\|\nabla^{3} \mathrm{u}\right\|_{2}^{2}
\end{aligned}
$$

where we have applied the inequality $\left\|\nabla^{2} u\right\|_{4} \leqslant\left\|\nabla^{2} u\right\|_{2}^{\frac{1}{4}}\left\|\nabla^{3} u\right\|_{2}^{\frac{3}{4}}$. Applying (3.1), the second term $\mathrm{J}_{2}$ can be estimated as

$$
\begin{aligned}
\mathrm{J}_{2} & =-\int_{\mathbb{R}^{3}} \nabla(\nabla \mathrm{d} \cdot \Delta \mathrm{d}) \cdot \nabla^{3} \mathrm{udx} \\
& \leqslant \mathrm{C} \int_{\mathbb{R}^{3}}\left|\nabla^{2} \mathrm{~d}\right|^{2}\left|\nabla^{3} \mathrm{u}\right| \mathrm{d} x+\int_{\mathbb{R}^{3}}\left|\nabla \mathrm{d}\|\nabla \Delta \mathrm{d}\| \nabla^{3} \mathrm{u}\right| \mathrm{d} x \\
& \leqslant \mathrm{C}\left\|\nabla^{2} \mathrm{~d}\right\|_{4}^{2}\left\|\nabla^{3} \mathrm{u}\right\|_{2}+\|\nabla \mathrm{d}\|_{4}\|\nabla \Delta \mathrm{d}\|_{4}\left\|\nabla^{3} \mathrm{u}\right\|_{2} \\
& \leqslant \mathrm{C}\left(\left\|\nabla^{2} \mathrm{~d}\right\|_{4}^{4}+\|\nabla \mathrm{d}\|_{4}^{2}\|\nabla \Delta \mathrm{d}\|_{4}^{2}\right)+\frac{1}{6}\left\|\nabla^{3} \mathrm{u}\right\|_{2}^{2} \\
& \leqslant \mathrm{C}\left(\left\|\nabla^{2} \mathrm{~d}\right\|_{2}^{\frac{5}{2}}\left\|\nabla^{4} \mathrm{~d}\right\|_{2}^{\frac{3}{2}}+\|\nabla \mathrm{d}\|_{2}^{\frac{1}{2}}\left\|\nabla^{2} \mathrm{~d}\right\|_{2}^{\frac{3}{2}}\left\|\nabla^{3} \mathrm{~d}\right\|_{2}^{\frac{1}{2}}\left\|\nabla^{4} \mathrm{~d}\right\|_{2}^{\frac{3}{2}}\right)+\frac{1}{6}\left\|\nabla^{3} \mathrm{u}\right\|_{2}^{2} \\
& \leqslant \mathrm{C}\left(\left\|\nabla^{2} \mathrm{~d}\right\|_{2}^{10}+\left\|\nabla^{2} \mathrm{~d}\right\|_{2}^{6}\left\|\nabla^{3} \mathrm{~d}\right\|_{2}^{2}\right)+\frac{1}{6}\left\|\nabla^{4} \mathrm{~d}\right\|_{2}^{2}+\frac{1}{6}\left\|\nabla^{3} \mathrm{u}\right\|_{2}^{2},
\end{aligned}
$$

where we have used the following Sobolev interpolation inequalities:

$$
\left\|\nabla^{2} \mathrm{~d}\right\|_{4}^{4} \leqslant\left\|\nabla^{2} \mathrm{~d}\right\|_{2}^{\frac{5}{2}}\left\|\nabla^{4} \mathrm{~d}\right\|_{2}^{\frac{3}{2}}, \quad\left\|\nabla^{3} \mathrm{~d}\right\|_{4}^{2} \leqslant\left\|\nabla^{3} \mathrm{~d}\right\|_{2}^{\frac{1}{2}}\left\|\nabla^{4} \mathrm{~d}\right\|_{2}^{\frac{3}{2}} .
$$

Again, with the aid of the commutator estimate (2.3), Young inequality and Hölder inequality, we may conclude that

$$
\begin{aligned}
\mathrm{J}_{3} & =-\int_{\mathbb{R}^{3}}\left[\nabla^{3}(\mathrm{u} \cdot \nabla \mathrm{d})-\mathrm{u} \cdot \nabla \nabla^{3} \mathrm{~d}\right] \nabla^{3} \mathrm{~d} \mathrm{~d} x \\
& \leqslant \mathrm{C}\|\nabla \mathrm{u}\|_{2}\left\|\nabla^{3} \mathrm{~d}\right\|_{4}^{2}+\left\|\nabla^{3} \mathrm{u}\right\|_{2}\|\nabla \mathrm{d}\|_{4}\left\|\nabla^{3} \mathrm{~d}\right\|_{4} \\
& \leqslant \mathrm{C}\|\nabla \mathrm{u}\|_{2}\left\|\nabla^{3} \mathrm{~d}\right\|_{2}^{\frac{1}{2}}\left\|\nabla^{4} \mathrm{~d}\right\|_{2}^{\frac{3}{2}}+\frac{1}{6}\left\|\nabla^{3} \mathrm{u}\right\|_{2}^{2}+\|\nabla \mathrm{d}\|_{4}^{2}\left\|\nabla^{3} \mathrm{~d}\right\|_{4}^{2} \\
& \leqslant \mathrm{C}\|\nabla \mathrm{u}\|_{2}^{4}\left\|\nabla^{3} \mathrm{~d}\right\|_{2}^{2}+\frac{1}{12}\left\|\nabla^{4} \mathrm{~d}\right\|_{2}^{2}+\frac{1}{6}\left\|\nabla^{3} \mathrm{u}\right\|_{2}^{2}+\mathrm{C}\left\|\nabla^{2} \mathrm{~d}\right\|_{2}^{6}\left\|\nabla^{3} \mathrm{~d}\right\|_{2}^{2}+\frac{1}{12}\left\|\nabla^{4} \mathrm{~d}\right\|_{2}^{2} \\
& \leqslant \mathrm{C}\left(\|\nabla \mathrm{u}\|_{2}^{4}+\left\|\nabla^{2} \mathrm{~d}\right\|_{2}^{6}\right)\left(\left\|\nabla^{2} \mathrm{u}\right\|_{2}^{2}+\left\|\nabla^{3} \mathrm{~d}\right\|_{2}^{2}\right)+\frac{1}{6}\left\|\nabla^{3} \mathrm{u}\right\|_{2}^{2}+\frac{1}{6}\left\|\nabla^{4} \mathrm{~d}\right\|_{2}^{2},
\end{aligned}
$$

where we have used (3.14) and the following Sobolev interpolation inequality:

$$
\|\nabla \mathrm{d}\|_{4} \leqslant\|\nabla \mathrm{d}\|_{2}^{\frac{1}{4}}\left\|\nabla^{2} \mathrm{~d}\right\|_{2}^{\frac{3}{4}}
$$

Finally, we move to estimate the last term $J_{4}$, by using the facts that $|d|=1$ and $|\nabla d|^{2}=-d \cdot \Delta d$, we obtain

$$
\begin{aligned}
\mathrm{J}_{4} & =-\int_{\mathbb{R}^{3}} \Delta\left(|\nabla \mathrm{d}|^{2} \mathrm{~d}\right) \cdot \Delta^{2} \mathrm{dd} x \\
& =-\int_{\mathbb{R}^{3}}\left[\Delta\left(|\nabla \mathrm{d}|^{2}\right) \mathrm{d}+2 \nabla\left(|\nabla \mathrm{d}|^{2}\right) \nabla \mathrm{d}+|\nabla \mathrm{d}|^{2} \Delta \mathrm{d}\right] \cdot \Delta^{2} \mathrm{~d} \mathrm{~d} x \\
& \leqslant\left\{\mathrm{C}\left(\left\|\left|\nabla^{2} \mathrm{~d}\right|^{2}\right\|_{2}+\left\|\nabla \mathrm{d} \cdot \nabla^{3} \mathrm{~d}\right\|_{2}+\left\||\nabla \mathrm{d}|^{2} \cdot\left|\nabla^{2} \mathrm{~d}\right|\right\|_{2}\right)+\left\|\mathrm{d}|\Delta \mathrm{d}|^{2}\right\|_{2}\right\} \cdot\left\|\Delta^{2} \mathrm{~d}\right\|_{2} \\
& \leqslant \mathrm{C}\left(\|\nabla \mathrm{d}\|_{4}\left\|\nabla^{3} \mathrm{~d}\right\|_{4}+\left\|\nabla^{2} \mathrm{~d}\right\|_{4}^{2}\right)\left\|\nabla^{4} \mathrm{~d}\right\|_{2} \\
& \leqslant \mathrm{C}\left(\|\nabla \mathrm{d}\|_{2}^{\frac{1}{2}}\left\|\nabla^{2} \mathrm{~d}\right\|_{2}^{\frac{3}{4}}\left\|\nabla^{3} \mathrm{~d}\right\|_{2}^{\frac{1}{4}}\left\|\nabla^{4} \mathrm{~d}\right\|_{2}^{\frac{3}{4}}+\left\|\nabla^{2} \mathrm{~d}\right\|_{2}^{\frac{5}{4}}\left\|\nabla^{4} \mathrm{~d}\right\|_{2}^{\frac{3}{4}}\right)\left\|\nabla^{4} \mathrm{~d}\right\|_{2} \\
& \leqslant \mathrm{C}\left(\left\|\nabla^{2} \mathrm{~d}\right\|_{2}^{6}\left\|\nabla^{3} \mathrm{~d}\right\|_{2}^{2}+\left\|\nabla^{2} \mathrm{~d}\right\|_{2}^{10}\right)+\frac{1}{6}\left\|\nabla^{4} \mathrm{~d}\right\|_{2}^{2} .
\end{aligned}
$$


Inserting (3.12)-(3.13) and (3.15)-(3.17) into (3.11), it yields that

$$
\begin{gathered}
\frac{1}{2} \frac{\mathrm{d}}{\mathrm{dt}}\left(\left\|\nabla^{2} \mathrm{u}\right\|_{2}^{2}+\left\|\nabla^{3} \mathrm{~d}\right\|_{2}^{2}\right)+\left\|\nabla^{3} \mathrm{u}\right\|_{2}^{2}+\left\|\nabla^{4} \mathrm{~d}\right\|_{2}^{2} \leqslant \\
\mathrm{C}\left(\|\nabla \mathrm{u}\|_{2}^{4}+\left\|\nabla^{2} \mathrm{~d}\right\|_{2}^{6}\right)\left(\left\|\nabla^{2} \mathrm{u}\right\|_{2}^{2}+\left\|\nabla^{3} \mathrm{~d}\right\|_{2}^{2}\right)+\mathrm{C}\left\|\nabla^{2} \mathrm{~d}\right\|_{2}^{10} \\
+\frac{1}{2}\left\|\nabla^{3} \mathrm{u}\right\|_{2}^{2}+\frac{1}{2}\left\|\nabla^{4} \mathrm{~d}\right\|_{2}^{2}
\end{gathered}
$$

Hence, by the Gronwall's inequality, we have

$$
\begin{aligned}
& \left(\left\|\nabla^{2} \mathrm{u}\right\|_{2}^{2}+\left\|\nabla^{3} \mathrm{~d}\right\|_{2}^{2}\right)+\int_{0}^{\mathrm{T}}\left(\left\|\nabla^{3} \mathrm{u}\right\|_{2}^{2}+\left\|\nabla^{4} \mathrm{~d}\right\|_{2}^{2}\right) \mathrm{dt} \\
& \quad \leqslant\left\{\left\|\nabla^{2} \mathrm{u}_{0}\right\|_{2}^{2}+\left\|\nabla^{3} \mathrm{~d}_{0}\right\|_{2}^{2}+\mathrm{C} \int_{0}^{\mathrm{T}}\left\|\nabla^{2} \mathrm{~d}\right\|_{2}^{10} \mathrm{dt}\right\} \exp \left\{\mathrm{T} \sup _{0<\mathrm{t}<\mathrm{T}}\|\nabla \mathrm{u}(\mathrm{t})\|_{2}^{4}+\left\|\nabla^{2} \mathrm{~d}(\mathrm{t})\right\|_{2}^{6}\right\} .
\end{aligned}
$$

By using the Sobolev embedding $\mathrm{H}^{2} \hookrightarrow \mathrm{L}^{\infty}\left(\mathbb{R}^{3}\right)$, we can obtain the BKM's criterion (1.3) immediately, which completes the proof of Theorem 1.1.

\section{Acknowledgment}

The research of B Yuan was partially supported by the National Natural Science Foundation of China (No. 11471103).

\section{References}

[1] H. Bahouri, J.-Y. Chemin, R. Danchin, Fourier analysis and nonlinear partial differential equations, Grundlehren der Mathematischen Wissenschaften [Fundamental Principles of Mathematical Sciences], Springer, Heidelberg, (2011). $1,2.1$

[2] K.-C. Chang, W. Y. Ding, R.-G. Ye, Finite-time blow-up of the heat flow of harmonic maps from surfaces, J. Differential Geom., 36 (1992), 507-515. 1

[3] Y. M. Chen, M. Struwe, Existence and partial regularity results for the heat flow for harmonic maps, Math. Z., 201 (1989), 83-103. 1

[4] D. Coutand, S. Shkoller, Well-posedness of the full Ericksen-Leslie model of nematic liquid crystals, C. R. Acad. Sci. Paris Sér. I Math., 333 (2001), 919-924. 1

[5] J. L. Ericksen, Hydrostatic theory of liquid crystals, Arch. Rational Mech. Anal., 9 (1962), 371-378. 1

[6] J.-S. Fan, Y. Zhou, Regularity criteria for the wave map and related systems, Electron. J. Differential Equations, 2016 (2016), 9 pages. 1

[7] S. Gala, Q. Liu, M. A. Ragusa, Logarithmically improved regularity criterion for the nematic liquid crystal flows in $\dot{\mathrm{B}}_{\infty, \infty}^{-1}$ space, Comput. Math. Appl., 65 (2013), 1738-1745. 1

[8] Y. Giga, Solutions for semilinear parabolic equations in $\mathrm{L}^{\mathrm{p}}$ and regularity of weak solutions of the Navier-Stokes system, J. Differential Equations, 62 (1986), 186-212. 1

[9] J. L. Hineman, C.-Y. Wang, Well-posedness of nematic liquid crystal flow in $\mathrm{L}_{\mathrm{uloc}}^{3}\left(\mathbb{R}^{3}\right)$, Arch. Ration. Mech. Anal., 210 (2013), 177-218. 1

[10] X.-P. Hu, D.-H. Wang, Global solution to the three-dimensional incompressible flow of liquid crystals, Comm. Math. Phys., 296 (2010), 861-880.

[11] T. Huang, C.-Y. Wang, Blow up criterion for nematic liquid crystal flows, Comm. Partial Differential Equations, 37 (2012), 875-884. 1, 1, 1

[12] T. Kato, G. Ponce, Commutator estimates and the Euler and Navier-Stokes equations, Comm. Pure Appl. Math., 41 (1988), 891-907. 2.2

[13] H. Kozono, Y. Taniuchi, Bilinear estimates in BMO and the Navier-Stokes equations, Math. Z., 235 (2000), 173-194. 1

[14] P. G. Lemarié-Rieusset, Recent developments in the Navier-Stokes problem, Chapman \& Hall/CRC Research Notes in Mathematics, Chapman \& Hall/CRC, Boca Raton, FL, (2002). 1

[15] J. Leray, Sur le mouvement d'un liquide visqueux emplissant l'espace, (French) Acta Math., 63 (1934), $193-248$.

[16] F. Leslie, Theory of flow phenomenum in liquid crystals, Springer, New York, (1979). 1

[17] X.-L. Li, D.-H. Wang, Global solution to the incompressible flow of liquid crystals, J. Differential Equations, 252 (2012), 745-767. 1

[18] F.-H. Lin, Nonlinear theory of defects in nematic liquid crystals; phase transition and flow phenomena, Comm. Pure Appl. Math., 42 (1989), 789-814. 1 
[19] J.-Y. Lin, S.-J. Ding, On the well-posedness for the heat flow of harmonic maps and the hydrodynamic flow of nematic liquid crystals in critical spaces, Math. Methods Appl. Sci., 35 (2012), 158-173. 1

[20] F.-H. Lin, J.-Y. Lin, C.-Y. Wang, Liquid crystal flows in two dimensions, Arch. Ration. Mech. Anal., 197 (2010), $297-336$. 1

[21] F.-H. Lin, C. Liu, Nonparabolic dissipative systems modeling the flow of liquid crystals, Comm. Pure Appl. Math., 48 (1995), 501-537. 1

[22] F.-H. Lin, C. Liu, Existence of solutions for the Ericksen-Leslie system, Arch. Ration. Mech. Anal., 154 (2000), 135-156. 1

[23] F.-H. Lin, C.-Y. Wang, On the uniqueness of heat flow of harmonic maps and hydrodynamic flow of nematic liquid crystals, Chin. Ann. Math. Ser. B , 31 (2010), 921-938. 1

[24] Q. Liu, J.-H. Zhao, A regularity criterion for the solution of nematic liquid crystal flows in terms of the $\dot{\mathrm{B}}_{\infty, \infty}^{-1}-$ norm, J. Math. Anal. Appl., 407 (2013), 557-566. 1

[25] Q. Liu, J.-H. Zhao, Logarithmically improved blow-up criteria for the nematic liquid crystal flows, Nonlinear Anal. Real World Appl., 16 (2014), 178-190. 1

[26] H. Sun, C. Liu, On energetic variational approaches in modeling the nematic liquid crystal flows, Discrete Contin. Dyn. Syst., 23 (2009), 455-475. 1

[27] C.-Y. Wang, Heat flow of harmonic maps whose gradients belong to $\mathrm{L}_{\chi}^{n} \mathrm{~L}_{t}^{\infty}$, Arch. Ration. Mech. Anal., 188 (2008), 351-369. 1

[28] C.-Y. Wang, Well-posedness for the heat flow of harmonic maps and the liquid crystal flow with rough initial data, Arch. Ration. Mech. Anal., 200 (2011), 1-19. 1

[29] H.-Y. Wen, S.-J. Ding, Solutions of incompressible hydrodynamic flow of liquid crystals, Nonlinear Anal. Real World Appl., 12 (2011), 1510-1531. 1

[30] J.-H. Zhao, BKM's criterion for the 3D nematic liquid crystal flows via two velocity components and molecular orientations, Math. Methods Appl. Sci., 40 (2017), 871-882. 1 